\title{
When is acute persistent cough in school-age children and adults whooping cough?
}

\author{
A prospective case series study
}

\begin{abstract}
\section{Background}

Pertussis is a vaccine modified disease in most age groups and hence subtle in its presentation. Current diagnostic approaches require relatively invasive sampling.
\end{abstract}

\section{Aim}

To determine the incidence of $B$. pertussis infection among people aged $5-49$ years identified in primary care with acute persistent cough using an oral fluid based diagnostic test.

\section{Design and setting}

Active surveillance of acute persistent cough of 2 weeks duration or greater was established in Auckland, New Zealand from May to October 2011. The 15 participating primary care practices provided care for a socioeconomically diverse population

\section{Method}

Recent $B$. pertussis infection was determined by measurement of $\lg G$ antibodies to pertussis toxin (PT) in an oral fluid sample. An lgG antibody titre to PT of $\geq 70$ arbitrary units defined recent infection. Participants reported symptoms at presentation and kept a cough diary.

\section{Results}

A total of 226 participants were enrolled: 70 (31\%) were children (5-16 years) and 156 (69\%) were adults (17-49 years). Oral fluid samples were obtained from 225 participants. Ten per cent (23/225) had recent B. pertussis infection including a larger proportion of children than adults ( $17 \%$ versus $7 \%, P=0.003)$. Neither cough duration nor any individual symptom discriminated between those with and without recent $B$. pertussis infection.

\section{Conclusion}

Pertussis is a frequent cause of acute persistent cough presenting to primary care. Clinical differentiation of pertussis from other causes of acute persistent cough is difficult. An oral fluid based diagnostic test, which is less invasive than other diagnostic approaches, has high acceptability in primary care.

\section{Keywords}

Bordetella pertussis; cough, general practice; signs and symptoms, respiratory; whooping cough.

\section{INTRODUCTION}

Pertussis is a highly contagious infection. Household contacts (people visiting or living in the home), are the most frequent source of infection for infants hospitalised with pertussis. ${ }^{1}$ Pertussis can be difficult to distinguish from other coughing illnesses, and this may be confounded in individuals who have previously received pertussis vaccination and have a more subtle presentation.

Studies of adolescents and adults with prolonged cough illnesses from Asia, Australia, Europe, and North America have shown that $B$. pertussis infection is a frequent cause of such illnesses. ${ }^{2}$ The ability to demonstrate recent infection with $B$. pertussis in these age groups was made possible by development of sensitive and specific ELISA assays that measure antibodies to $B$. pertussis antigens in sera. Initially paired serum samples were necessary but more recently, diagnosis based upon measurement of $\lg G$ antibodies to pertussis toxin (PT) on a single serum sample has become possible. ${ }^{3}$ However requiring a blood sample for diagnosis reduces the acceptability of such testing. particularly for children.

Acute persistent cough is a common presentation in general practice with an extensive differential diagnosis. Diagnostic uncertainty potentially leads to excessive investigation and management. Being able

K Philipson, MBChB, Master in Public Health student, Department of General Practice \& Primary Health Care; $\mathbf{F}$ Goodyear-Smith, MD, FRNZCGP, GP and professor in the Department of General Practice \& Primary Health Care; cc Grant, PhD, FACP, paediatrician and associate professor in paediatrics; A Chong, BBus, RGN, research nurse and project manager; $\mathbf{N}$ Turner, MPH, FRNZCGP, GP and director, Immunisation Advisory Centre; J Stewart, MSc, senior research fellow and biostatistician, Faculty of Medical

\& Health Sciences, University of Auckland, Auckland, New Zealand. to establish the presence of $B$. pertussis infection is therefore not only important from an infection control perspective but also reduces unnecessary further intervention. Ability to achieve this in primary care has been restricted by the limited availability of diagnostic tests with adequate sensitivity and specificity and with a simple and non-invasive collection of the biological sample.

The aim of this study was to estimate the proportion of school-aged children and adults under 50 years of age identified in general practice with acute persistent cough who had recent infection with $B$. pertussis and to determine whether there are symptoms that predict $B$. pertussis infection. The study used an oral fluidbased assay as the diagnostic test for $B$. pertussis infection, and to demonstrate the applicability of this test to the primary care setting.

\section{METHOD}

\section{Study design and setting}

The study completed a prospective case series of school-age children laged 5-16 years) and adults (17-49 years) identified in primary care in Auckland, New Zealand, with an acute persistent cough of 2 weeks duration or greater between May and October 2011. Cases were identified from a convenience sample of general practices, selecting for socioeconomic

\section{Address for correspondence}

Felicity Goodyear-Smith, Department of General Practice \& Primary Health Care, University of Auckland, PB 92019 Auckland, New Zealand. E-mail: f.goodyear-smithdauckland.ac.nz Submitted: 19 January 2013; Editor's response: 28 February 2013; final acceptance: 25 March 2013.

\section{CBritish Journal of General Practice}

This is the full-length article (published online 29 Jul 2013) of an abridged version published in print. Cite this article as: $\mathbf{B r} \mathbf{J}$ Gen Pract 2013; DOI: 10.3399/bjgp13X670705 


\section{How this fits in}

Pertussis is a recognised cause of acute persistent cough presenting to primary care in first world countries. Laboratory diagnosis of pertussis in the primary care setting is difficult with the clinical presentation of pertussis to primary care in populations that have received booster doses of acellular pertussis vaccine not well defined. In this study it is demonstrated that an oral fluid based assay that measures antibodies to pertussis toxin is an ideal test for the primary care setting. In New Zealand, where pertussis vaccine boosters are given at ages 4 and 11 years, recent Bordetella pertussis infection is evident in $17 \%$ of children $5-16$ years old and $7 \%$ of adults 17-49 years old identified in primary care with acute persistent cough. Neither cough duration nor any individual presenting symptom discriminate those with pertussis from those with acute persistent cough due to other causes.

diversity and pragmatic factors including willingness to engage in primary care research.

\section{Participants}

The study sought to enrol 200 children and adults aged 5-49 years with an acute persistent cough. The cut-off age of 49 years was a pragmatic decision to enable comparison with a similar study being conducted by the study's Oxford colleagues. The study excluded those with an underlying medical condition which may cause a persistent cough (for example, bronchiectasis, cardiac failure) or who were immune-compromised. A number of

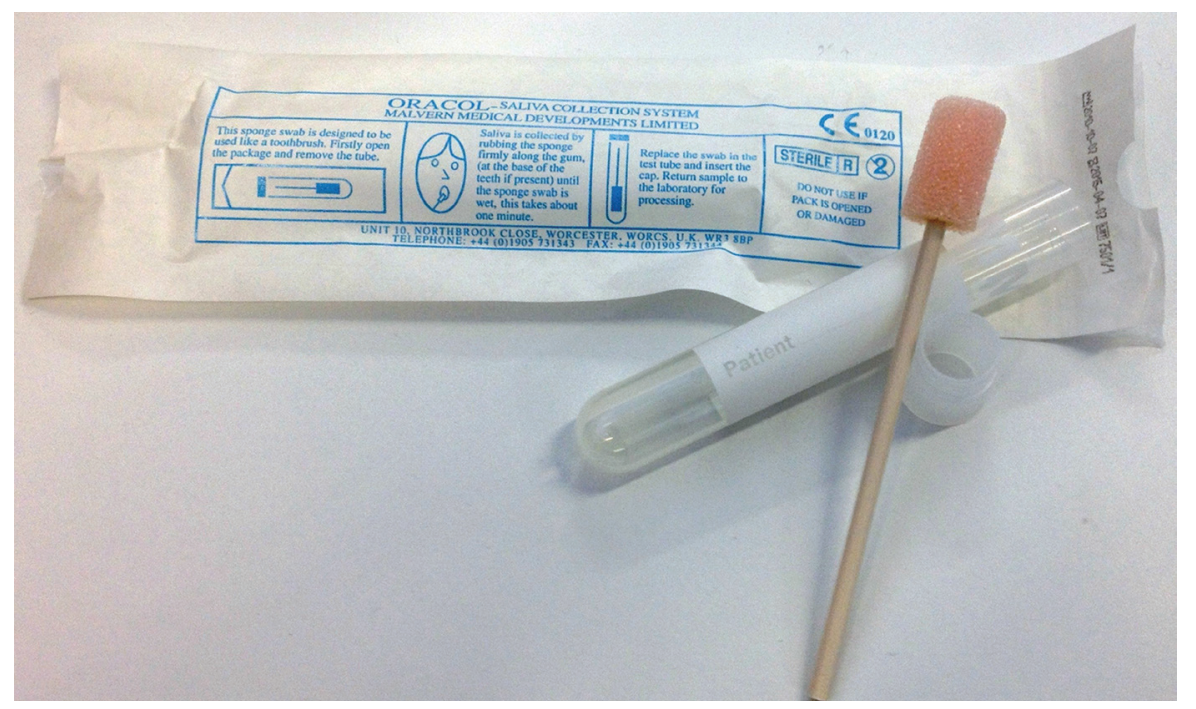

studies have found an incidence of pertussis in primary care and emergency department settings in the range $10-30 \% .^{4-9} \mathrm{~A}$ sample size of 200 was sufficient to estimate the incidence of $B$. pertussis infection with $95 \%$ confidence limits of $\pm 4 \%$ if the true incidence was $10 \%$, and $\pm 6 \%$ if the true incidence was $30 \%$.

\section{Recruitment}

Potential participants were invited to participate by members of the general practice team (GP, practice nurse, administration staff). Details of eligible patients who agreed to participate were sent by practice staff to the research nurse, who then recruited participants for the study.

During a face-to-face interview written informed consent was obtained and participants completed a questionnaire, self-administered collection of an oral fluid (Oracol) swab and agreed to complete a cough diary. Collection of the oral fluid sample involved rubbing the applicator sponge over the gums for 1 minute, hence is a sample of gingival fluid rather than saliva. A daily cough diary was kept for 14 days, with a further weekly diary for 12 weeks if their cough persisted, or until the cough stopped.

\section{Diagnosis of $\boldsymbol{B}$. pertussis infection}

Recent $B$. pertussis infection was determined by measurement of the concentration of $\lg G$ antibodies to pertussis toxin (PT) in the oral fluid sample. While previously a significant increase in paired sera samples was considered necessary for diagnosis, studies testing serum specimens vaccinees and pertussis patients have found high IgG levels to be very rare in the general population. ${ }^{10}$ IgG antibodies appear in the early weeks after infection, peak within a few months, and then decline. A study using matched serum and oral fluid samples in children whose serum anti-PT titre had already been determined, found that the oral fluid assay detected seropositive subjects with a sensitivity of $80 \%$ and a specificity of $97 \% .{ }^{11}$ A single oral fluid assay is therefore a valid surrogate for a serum ELISA.

Currently this assay is only measurable at the Respiratory and Systemic Infection Laboratory (RSIL), England. To maintain sample integrity samples were kept chilled using ice packs and/or refrigeration. LabPLUS pre-processed samples, eluted from the Oracol swab (Figure 1) using a transport medium. Once eluted the contents were split into two microtubes, one as a master and one as a backup and 
Figure 2. Summary of patient enrolment and data collection.

\section{Table 1. Characteristics of the 226 enrolled participants}

\begin{tabular}{|c|c|}
\hline Characteristic & $n(\%)$ \\
\hline \multicolumn{2}{|l|}{ Age, years } \\
\hline $5-16$ & 70 (31) \\
\hline $17-49$ & $156(69)$ \\
\hline Female sex & $143(63)$ \\
\hline \multicolumn{2}{|l|}{ Ethnicity } \\
\hline European & $59(26)$ \\
\hline Maori & $35(15)$ \\
\hline Pacific ${ }^{b}$ & $36(16)$ \\
\hline Asianc & $71(31)$ \\
\hline Other & $25(11)$ \\
\hline \multicolumn{2}{|l|}{ Education ${ }^{a}$} \\
\hline Did not complete secondary & $23(10)$ \\
\hline Secondary & $31(14)$ \\
\hline Tertiary & 170 (75) \\
\hline \multicolumn{2}{|l|}{ Smokers in household } \\
\hline 0 & 145 (64) \\
\hline 1 & $46(20)$ \\
\hline$\geq 2$ & $35(15)$ \\
\hline \multicolumn{2}{|l|}{ Household size } \\
\hline $1-2$ & $26(12)$ \\
\hline $3-4$ & $91(40)$ \\
\hline $5-9$ & $98(43)$ \\
\hline$\geq 10$ & $11(5)$ \\
\hline \multicolumn{2}{|c|}{${ }^{a}$ Of mother for enrolled children. ${ }^{b} / n c l u d e s$} \\
\hline \multicolumn{2}{|c|}{ Samoan $(n=16)$, Tongan $(n=7)$, Nivean $(n=5)$, } \\
\hline \multicolumn{2}{|c|}{ Cook Islander ( $\mathrm{n}=5$ ), Tuvalu (2), Fijian ( $\mathrm{n}=1$ ). } \\
\hline \multicolumn{2}{|c|}{ c/ncludes Chinese $(n=16)$, Indian $(n=29)$, Fijian } \\
\hline \multicolumn{2}{|c|}{ Indian ( $\mathrm{n}=9$ ), Filipino ( $\mathrm{n}=9$ ), Indonesian ( $\mathrm{n}=2$ ), } \\
\hline \multicolumn{2}{|c|}{ Korean $(\mathrm{n}=3)$, Sri Lankan $(\mathrm{n}=1)$, Thai $(\mathrm{n}=1)$, } \\
\hline Cambodian (n = 1). & \\
\hline
\end{tabular}

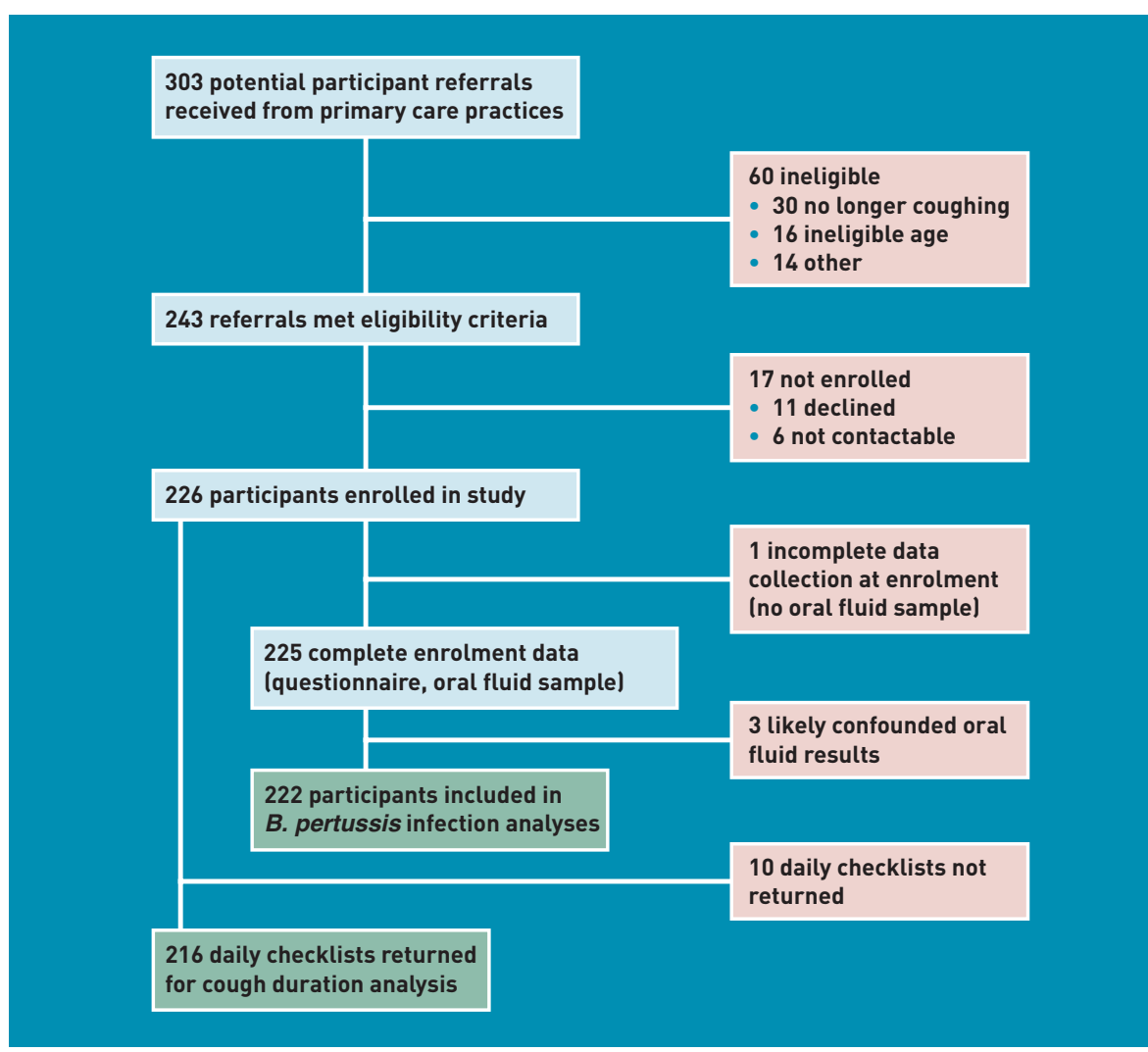

then stored at $-20^{\circ} \mathrm{C}$. The two samples were stored separately to minimise risks of thawing during handling. Samples were air-freighted in two batches. Serial readings on the waybills recorded that the sample temperature was between -60 to $-80^{\circ} \mathrm{C}$ throughout the transportation period. RSIL reported that all Oracol samples received were of gingival fluid not saliva and were of adequate quality to undergo the specific PT $\lg G$ antibody assay.

\section{Data analysis}

Descriptive statistics, Cox proportional hazards survival analysis with Kaplan-Meier survival plots, logistic regression were performed using PC-SAS (SAS version 9.2) and a classification analysis was performed using SPAN (Search Partition Analysis Software) SPAN uses Boolean expressions to best partition the data to predict an outcome. ${ }^{12}$

\section{RESULTS}

\section{Sample characteristics}

Fifteen practices from a range of socioeconomic positions were recruited. Participants were referred predominantly by nurses and receptionists but also by GPs. Of the 303 potential participant referrals, 243 (80\%) met study eligibility criteria. Reasons for ineligibility were no longer coughing, outside the age range, had not presented to the practice, on immunosuppressive therapy, or cough defined as normal for them'. Because of the nature of general practice there are likely to be a number of other patients who were potentially eligible (that is, had 2 weeks of cough) who were not invited by practice staff to participate.

Of 243 eligible participants, 226 (93\%) were enrolled. Eleven declined and questionnaires were completed by $100 \%$. Oral fluid samples were obtained from 225 (99.5\%) (one child was too ill for a sample to be obtained). Two hundred and sixteen (96\%) returned the daily cough checklist, and $90 \%$ of required weekly checklists were received (Figure 2).

Seventy $(31 \%)$ of the 226 participants were children (5-16 years) and 156 (69\%) were adults (17-49). Approximately equal proportions of the 70 enrolled children were boys or girls ( $49 \%$ versus $51 \%$ ). A larger proportion of the 156 enrolled adults were female Ifemale versus male, $69 \%$ versus $31 \%)$. The sample was ethnically diverse (Table 1). Eighty per cent of adults selfreported previous pertussis vaccination although only $2 \%$ could verify the date of their last pertussis vaccine.

Of the 156 adult participants, 22\% worked in high contact, at-risk roles for $B$. pertussis six were unable to be contacted. Study 


\section{Table 2. Positive oral fluid Pertussis Toxin IgG assay}

\begin{tabular}{|c|c|c|c|c|c|}
\hline \multirow{2}{*}{$\begin{array}{l}\text { Study ID } \\
\text { Children }\end{array}$} & \multirow[t]{2}{*}{ Sex } & \multicolumn{2}{|c|}{ Age, years months } & \multirow[t]{2}{*}{ Sample date } & \multirow[t]{2}{*}{$\begin{array}{c}\text { Pertussis } \\
\text { IgG titre, AU }\end{array}$} \\
\hline & & & & & \\
\hline CS105-C & Female & 11 & 3 & 13-Jun-11 & 159 \\
\hline CS103-C & Male & 11 & 4 & 3-Jul-11 & 83 \\
\hline CS107-C & Male & 13 & 8 & 3-Jul-11 & 290 \\
\hline CS121-C & Male & 5 & 9 & 19-Jul-11 & 267 \\
\hline CS122-C & Male & 15 & 8 & 22-Jul-11 & 86 \\
\hline CS138-C & Female & 10 & 3 & 29-Jul-11 & 223 \\
\hline CS142-C & Male & 15 & 11 & 11-Aug-11 & 372 \\
\hline CS144-C & Male & 14 & 9 & 11-Aug-11 & 90 \\
\hline CS134-C & Male & 5 & 4 & 23-Aug-11 & 238 \\
\hline CS148-C & Female & 9 & 0 & 29-Aug-11 & 80 \\
\hline CS149-C & Female & 5 & 7 & 30-Aug-11 & 131 \\
\hline CS154-C & Female & 12 & 1 & 1-Sep-11 & $62^{a}$ \\
\hline CS159-C & Male & 8 & 8 & 20-Sep-11 & 129 \\
\hline CS173-C & Male & 12 & 6 & 18-0ct-11 & 88 \\
\hline CS169-C & Female & 9 & 0 & $26-0 c t-11$ & 84 \\
\hline \multicolumn{6}{|l|}{ Adults } \\
\hline CS001-A & Female & 47 & 6 & 24-May-11 & 125 \\
\hline CS015-A & Female & 36 & 6 & 24-Jun-11 & 124 \\
\hline CS070-A & Female & 39 & 7 & 4-Aug-11 & 600 \\
\hline CS071-A & Male & 28 & 2 & 4-Aug-11 & 228 \\
\hline CS084-A & Male & 49 & 10 & 15-Aug-11 & 91 \\
\hline CS086-A & Male & 19 & 1 & 15-Aug-11 & 81 \\
\hline CS218-A & Female & 37 & 8 & 6-Sep-11 & 155 \\
\hline CS242-A & Female & 29 & 10 & 27-Sep-11 & 203 \\
\hline CS238-A & Male & 37 & 0 & 29-Sep-11 & 75 \\
\hline CS239-A & Male & 24 & 10 & 4-Oct-11 & 109 \\
\hline CS249-A & Female & 25 & 4 & $6-0 c t-11$ & 243 \\
\hline
\end{tabular}

${ }^{a}$ Borderline result, close to diagnostic threshold. $A U=$ arbitrary unit. the assay was consistent with recent $B$. pertussis infection with the oral fluid IgG to PT concentration ranging from 75 to $600 \mathrm{AU}$ (Table 2). One result (62 AU) was interpreted as having elevated antibodies close to the diagnostic threshold. Two children with a positive result and the child with the borderline result had received a dose of pertussis vaccine within the previous 12 months, potentially confounding their assay results. Excluding these three, recent $B$. pertussis infection was present in 23 of $223(10 \%)$ including $7 \%$ of adults (11/156; five male and six female) and $17 \%$ of children (12/66; seven male and five female).

The positive rate in children was higher than in adults, $P=0.003$ ). Five children with an elevated PT antibody titre had a symptomatic parent enrolled into the study who returned a negative result. Five of the 11 adults (45\%) with $B$. pertussis infection were working or studying in high contact, at risk roles for $B$. pertussis transmission.

\section{Cough duration and pertussis serology}

The number of days participants reported coughing could not be shown to differ between those with or without recent $B$. pertussis infection (Cox proportional hazard ratio $1.09,95 \% \mathrm{Cl}=0.71$ to $1.68, P=0.68$ ) (Figure 3).

\section{Symptoms associated with recent Bordetella pertussis infection}

Analysis of the children's data showed weak evidence for vomiting ( $P=0.08)$, but not wheezing $(P=0.05)$, and not headache $(P=0.07)$ to be associated with recent $B$. pertussis infection. In adults no individual symptoms could be shown to be associated with positive oral fluid PT assays.

In adults, the SPAN analysis found the best predictor of pertussis was the combined symptom clusters exercise intolerance, wheezing and vomiting, or exercise intolerance and wheezing but without headache or muscle pain Isensitivity and specificity for predicting recent $B$. pertussis infection were 0.73 , positive predictive value (PPV) 0.17 , negative predictive value (NPV) 0.97) (Table 3).

For children the best predictor was more complex: presence of vomiting and exercise intolerance but not wheezing or sneezing; or vomiting but not headache; or phlegm but not headache or wheeze; or phlegm and exercise intolerance but not wheeze or sneeze. When all of these symptom clusters were applied to the children's data the sensitivity for predicting recent $B$. pertussis infection was 0.67 and specificity 0.87, PPV 0.53, NPV 0.92 (Table 3). 
Table 3. Symptom clusters and recent Bordetella pertussis infection in adults and children

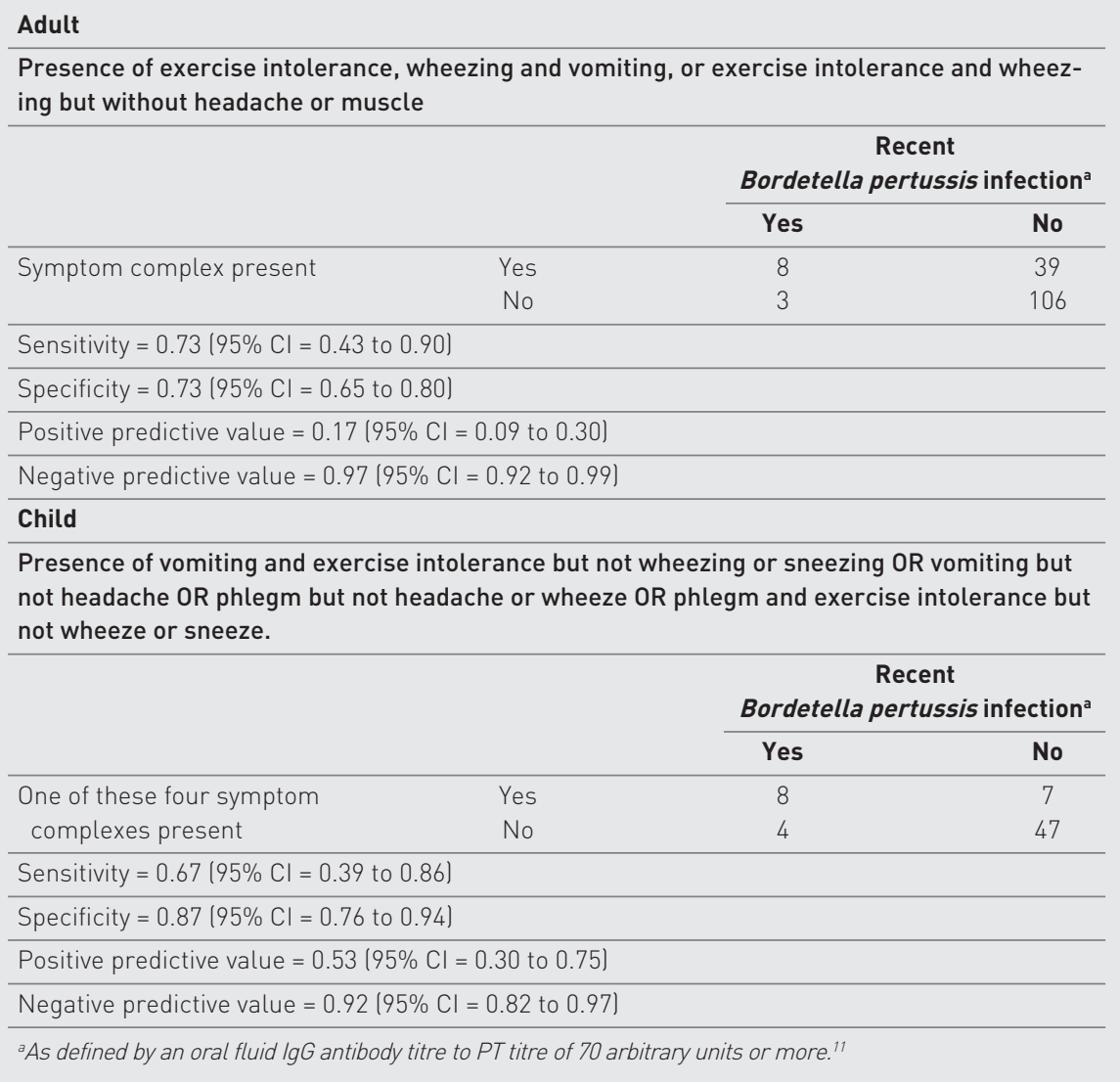

\section{DISCUSSION}

\section{Summary}

New Zealand is a country with a large infant pertussis disease burden. In the 2000s the New Zealand hospitalisation infant rate $(196 / 100000)^{13}$ was threefold higher than contemporary rates in the US (65/100 000), ${ }^{14}$ and Australia (56/100 000)..$^{15}$ The study indicates that $B$. pertussis infection is present in one in 10 individuals aged 5-49 years identified in primary care with acute persistent cough, with a larger proportion of children $(17 \%)$ than adults (7\%). Neither cough duration nor any individual presenting symptom differentiates $B$. pertussis infection from other causes of acute persistent cough.

\section{Strengths and limitations}

The study established active surveillance for acute persistent cough in a socioeconomically diverse group of general practices. The study maintained good retention of recruiting practices and a high enrolment and completion rate of patients identified with acute persistent cough. Use of the minimally invasive oral fluid swab enabled diagnostic testing to be completed on all but one of 226 participants. All oral fluid samples collected were of adequate quality for assay, $100 \%$ of enrolees completed the questionnaire and this study achieved high completion rates for the daily (96\%) and weekly (90\%) cough checklists.

The study was conducted over one winter season, therefore the estimate of the proportion of acute persistent cough that was secondary to $B$. pertussis infection may be lower than that which would have occurred with recruitment over the full calendar year. The seasonality of pertussis is less distinct than that of illnesses caused by respiratory viruses. Epidemic peaks of pertussis have been described in spring and summer (Australia), ${ }^{16}$ summer and autumn (The Netherlands) ${ }^{10}$ and in winter (Norway, Sweden). ${ }^{17}$ Thus by limiting the recruitment period as was done, the study have sampled from a portion of the year when respiratory viruses will have been the predominant infectious cause of acute persistent cough.

Patients could be referred by any member of the practice staff rather than solely the GP. In primary care-based studies of acute 
persistent cough that have relied solely on GP identification of cases, conducted as vaccine efficacy trials, a selection bias towards those with an illness more consistent with the classical descriptions of pertussis has been shown to sometimes occur. ${ }^{18,19}$ Thus the recruitment strategy may have yielded a truer estimate of the incidence of $B$. pertussis in those with acute persistent cough than would have been achieved if the study had restricted the enrolment to GP referrals.

\section{Comparison with existing literature}

Bordetella pertussis infection was identified less frequently in children in this study than it was in a primary care-based study from Oxfordshire which, using the same diagnostic test, identified pertussis in $37 \%$ of children 5-16 years old with acute persistent cough. ${ }^{8}$ Key differences between studies include the recruitment period (Oxfordshire 42 months versus Auckland 6 months), source of referrals (Oxfordshire GP only, Auckland any practice personnel) and differences in the immunisation schedule. In England over most of the time the Oxfordshire study was recruiting lOctober 2001 to March 2005) the schedule included a primary three dose whole-cell vaccine series alone, with the preschool acellular pertussis vaccine booster introduced only in $2004 .{ }^{20}$ With the waning of vaccine efficacy Ishown to be only approximately 50\% 5 years after immunisation with the British whole-cell vaccine) it is to be expected that a larger proportion of school aged children in England would have pertussis than schoolaged children in New Zealand where acellular pertussis vaccine booster doses are given at age 4 and 11 years ${ }^{21}$ following a three dose primary infant series. ${ }^{22}$

That the school-aged population in New Zealand received more booster vaccinations also explains the more clinically subtle presentation of pertussis in the study. In contrast to the study, longer cough duration, whoop, vomiting, and sputum production all predicted an increased likelihood of $B$. pertussis infection in England. ${ }^{8} \mathrm{~A}$ lack of difference in cough duration and clinical presentation between those with and without $B$. pertussis infection was recently reported from a French general practice-based survey of adolescents and adults with cough illness. ${ }^{23}$ France introduced an adolescent booster dose of pertussis vaccine in 1998 and a cocooning immunisation strategy in 2004.

\section{Implications for research and practice}

The frequency with which $B$. pertussis infection was identified in the study is consistent with frequencies reported in other cough studies of adolescents and adults $(12-52 \%)^{2}$ B. pertussis infection is a frequent cause of acute persistent cough irrespective of whether or not a pertussis outbreak is recognised to be present, hence pertussis should always be included in the differential diagnosis of acute persistent cough.

As the study showed, the clinical presentation is subtle and difficult to distinguish from other causes of acute persistent cough. There is a need for a diagnostic test to enable GPs to differentiate pertussis from other causes of acute persistent cough. The oral fluid-based test used in this study is ideal for the primary care setting given that its sensitivity and specificity were established in this environment, it is non-invasive, and easy to collect. At present though, the assay can be performed only at the RSIL laboratory. There is a need for an assay of this type to be more readily available to GPs.

Twenty-two per cent of the adults with acute persistent cough worked with children or in the health sector. The frequency of $B$. pertussis infection in this subgroup was three times higher than for other adults. This highlights the need both for access to high quality laboratory testing from primary care, and for the consideration of other immunisation strategies such as cocooning for population subgroups more likely to come in contact with young infants. ${ }^{24}$

The oral fluid assay creates the potential for the understanding of pertussis epidemiology to increase. Substantial under reporting of pertussis has been recognised for many decades and is one of the defining features of pertussis epidemiology. ${ }^{25}$ The availability of a diagnostic test which is non-invasive and has adequate sensitivity and specificity facilitates population-based studies of pertussis epidemiology that are less likely to be compromised by poor response rates or highly selected patient populations.

B. pertussis is a common cause of acute persistent cough in New Zealand schoolaged children and younger adults identified in primary care with acute persistent cough. In populations that have received booster immunisations, pertussis is difficult to distinguish clinically from other causes of acute persistent cough. An oral fluid-based assay is an ideal test for primary care settings and efforts should be made to make this test widely available. This would improve diagnosis and aid on-going refinement of pertussis immunisation policy. 


\section{REFERENCES}

1. Mclntyre P, Wood N. Pertussis in early infancy: disease burden and preventive strategies. Curr Opin Infect Dis 2009; 22(3): 215-223.

2. Cherry JD. The epidemiology of pertussis: a comparison of the epidemiology of the disease pertussis with the epidemiology of Bordetella pertussis infection. Pediatrics 2005; 115(5): 1422-1427.

3. de Melker HE, Versteegh FG, Conyn-Van Spaendonck MA, et al. Specificity and sensitivity of high levels of immunoglobulin $G$ antibodies against pertussis toxin in a single serum sample for diagnosis of infection with Bordetella pertussis. $J$ Clin Microbiol 2000; 38(2): 800-806.

4. Diez-Domingo J, Ballester A, Baldó JM, et al. Incidence of pertussis in persons $<$ or $=15$ years of age in Valencia, Spain: seroprevalence of antibodies to pertussis toxin (PT) in children, adolescents and adults. J Infect 2004; 49(3) 242-247.

5. Nennig ME, Shinefield HR, Edwards KM et al. Prevalence and incidence of adult pertussis in an urban population. JAMA 1996; 275(21): 1672-1674.

6. Wright SW, Edwards KM, Decker MD, Zeldin MH. Pertussis infection in adults with persistent cough. JAMA 1995; 273(13): 1044-1046.

7. Lasserre A, Laurent E, Turbelin C, et al. Pertussis incidence among adolescents and adults surveyed in general practices in the Paris area, France, May 2008 to March 2009. Euro Surveill 2011; 16(5). pii: 19783.

8. Harnden A, Grant C, Harrison T, et al. Whooping cough in school age children with persistent cough: prospective cohort study in primary care. BMJ 2006; 333(7560): $174-177$.

9. Gilberg S, Njamkepo E, Du Châtelet IP, et al. Evidence of Bordetella pertussis infection in adults presenting with persistent cough in a french area with very high whole-cell vaccine coverage. J Infect Dis 2002; 186(3): 415-418.

10. de Melker HE, Schellekens JF, Neppelenbroek SE, et al. Reemergence of pertussis in the highly vaccinated population of the Netherlands: observations on surveillance data. Emerg Infect Dis 2000; 6(4): 348-357.

11. Litt DJ, Samuel D. Duncan J, et al. Detection of anti-pertussis toxin IgG in oral fluids for use in diagnosis and surveillance of Bordetella pertussis infection in children and young adults. J Med Microbiol 2006; 55(Pt 9): 1223-1228.

12. Marshall RJ. Partitioning methods for classification and decision making in medicine. Stat Med 1986; 5(5): 517-526.

13. Grant $\mathrm{C}$. Recent indication of progress in pertussis hospitalisation rates in NZ Aust NZ J Pub Health 2012; 36(4): 398.

14. Cortese MM, Baughman AL, Zhang R, et al. Pertussis hospitalizations among infants in the United States, 1993 to 2004. Pediatrics 2008; 121(3): 484-492.

15. Elliott E, McIntyre P, Ridley G, et al. National study of infants hospitalized with pertussis in the acellular vaccine era. Pediatr Infect Dis J 2004; 23(3): 246-252.

16. Andrews R, Herceg A, Roberts C. Pertussis notifications in Australia, 1991 to 1997. Commun Dis Intell 1997; 21(11): 145-148.

17. Lindgren C, Milerad J, Lagercrantz H. Sudden infant death and prevalence of whooping cough in the Swedish and Norwegian communities. Eur J Pediatr 1997; 156(5): 405-409

18. Cherry JD, Heininger U, Stehr K, Christenson P. The effect of investigator compliance (observer bias) on calculated efficacy in a pertussis vaccine trial. Pediatrics 1998; 102(4 Pt 1): 909-912.

19. Stehr K, Cherry JD, Heininger $U$, et al. A comparative efficacy trial in Germany in infants who received either the Lederle/Takeda acellular pertussis component DTP (DTaP) vaccine, the Lederle whole-cell component DTP vaccine, or DT vaccine. Pediatrics 1998; 101(1 Pt 1): 1-11.

20. Health Protection Agency. Vaccine policy developments. Developments in vaccine policy and practice from 1990. London: Health Protection Agency, 2012

21. Jenkinson $D$. Duration of effectiveness of pertussis vaccine: evidence from a 10 year community study. Br Med J (Clin Res Ed) 1988; 296(6622): 612-614.

22. Ministry of Health. Immunisation handbook 2011. Wellington: Ministry of Health, 2011

23. Lasserre $\mathrm{A}$, Laurent $\mathrm{E}$, Turbelin $\mathrm{C}$, et al. Pertussis incidence among adolescents and adults surveyed in general practices in the Paris area, France, May 2008 to March 2009. Euro Surveill 2011 Feb 3;16(5). pii: 19783.

24. Forsyth KD, Wirsing von Konig C-H, Tan T, et al. Prevention of pertussis: recommendations derived from the second Global Pertussis Initiative roundtable meeting. Vaccine 2007; 25(14): 2634-2642.

25. Grant CC, Cherry JD. Keeping pace with the elusive Bordetella pertussis. J Infect 2002; 44(1): 7-12. 